\title{
LeGes
}

Giovanni Bruno

\section{Pericolo nel ritardo: agire immediatamente o senza indugio?}

Nella legislazione federale la tempistica con cui un atto va compiuto con una certa urgenza è di norma espressa mediante la locuzione avverbiale «senza indugio» («unverzüglich», «sans délai») e l'avverbio «immediatamente» («sofort», «immédiatement»). I termini non sono tuttavia intercambiabili e rispondono anzi a precise esigenze legate alle singole situazioni. Gli esempi mostrano che non sempre l'intervallo di tempo imposto o concesso è reso con l'espressione appropriata al caso specifico. Ma neanche i relativi testi esplicativi, come ad esempio i messaggi e i commentari, distinguono sempre adeguatamente tra le diverse situazioni.

Categoria di articoli: Contributi scientifici

Citazione: Giovanni Bruno, Pericolo nel ritardo: agire immediatamente o senza indugio?, in: LeGes $31(2020) 3$ 


\section{Indice}

1. Introduzione

2. L'imperativo di celerità tra Costituzione federale e procedura penale

3. Immediatezza e indugio nella legislazione federale

3.1. «unverzüglich»

3.1.1. Esempi conformi

3.1.2. Esempi non conformi

3.2. «sofort»

3.3. «umgehend»

4. Un caso particolare: «unverzüglich» nel CPP

4.1. Diversificazione terminologica nel CPP

4.2. La tempistica nei commentari al CPP

4.3. Il difficile rapporto tra legge e messaggio: l'articolo 209 capoverso 2 CPP

5. I difetti nascosti del Codice delle obbligazioni

6. L'indeterminatezza al vaglio delle autorità giudiziarie

6.1. Giurisprudenza federale: la celerità nel CO

6.2. Giurisprudenza cantonale: la celerità nel CPP

7. Conclusione

\section{Introduzione}

[1] Il brocardo del titolo, che corrisponde a «Gefahr im Verzug» e "péril en la demeure» nelle altre lingue ufficiali della legislazione svizzera, rispecchia l'esigenza del legislatore di non far ritardare l'esecuzione di determinati atti di varia natura. Per indicare un atto che va compiuto subito e un atto che può essere compiuto non subito ma comunque senza tardare occorre usare avverbi e locuzioni avverbiali che esplicitino in modo chiaro la tempistica imposta o concessa. Le espressioni usuali nella legislazione federale sono «immediatamente» e «subito» per un'esecuzione istantanea («sofort»e «immédiatement» nelle altre lingue della legislazione federale) e «senza indugio» per un'esecuzione non istantanea ma comunque senza ritardo («unverzüglich» e «sans délai», con alcune varianti). La seconda modalità di esecuzione può anche essere immediata se non vi sono motivi per differirla.

[2] Fra le espressioni menzionate quella più intrigante è sicuramente «senza indugio». Praticamente estranea al linguaggio comune, dove è percepita come una variante alta di «subito»e «immediatamente», ha invece assunto un suo significato ben preciso nel linguaggio giuridico. Il Vocabolario Treccani online, per esempio, sembra mettere le espressioni sullo stesso piano: «senza indugio, senza tardare, subito, immediatamente», mentre nella sezione dei Sinonimi e dei Contrari le espressioni sono date come (più o meno) sinonimiche: «Locuz. prep.: senza indugio all'istante, (lett., non com.) immantinente, immediatamente, istantaneamente, prontamente, senza por tempo in mezzo, subito.».

[3] Per quanto riguarda "subito» lo stesso vocabolario dà come sinonimi «all'istante, (fam.) detto fatto, (lett.) immantinente, immediatamente, ipso facto, senza indugio», mentre per «immediatamente», nella sua accezione temporale, riporta i sinonimi «all'istante, detto fatto, (lett.) immantinente, istantaneamente, senza por tempo in mezzo, subito, su due piedi».

[4] Sia «senza indugio» che «immediatamente» possono però avere anche valore non temporale. L'indugio può essere anche l'esitazione, il dubbio, e quindi «senza indugio», a seconda del contesto, può voler dire «senza ulteriori accertamenti», «senza chiedere pareri» e via dicendo, ossia in modo diretto e risoluto. L'avverbio «immediatamente» può invece indicare anche un'e- 
secuzione senza soluzione di continuità spaziale («senza l'interposizione di alcunché», secondo il Vocabolario Treccani).

[5] Dal canto suo il Duden-Online-Wörterbuch, per il linguaggio comune, definisce «unverzüglich» come «umgehend und ohne Zeitverzug [erfolgend]», mentre per «sofort» fornisce la definizione «ohne zeitliche Verzögerung; unverzüglich». Nel linguaggio giuridico tedesco, tuttavia, «unverzüglich» ha un significato equivalente all'espressione «senza indugio» della legislazione federale in italiano. A questo proposito la legislazione della Germania si trova in una condizione privilegiata in quanto dispone di una vera e propria definizione legale di questo termine. Il paragrafo 121 del Bürgerliches Gesetzbuch (BGB; stato 01.04.2020), il Codice civile tedesco, definisce di fatto, pur indirettamente, il concetto di «unverzüglich» (i corsivi nelle disposizioni citate in questo contributo sono sempre nostri):

«(1) Die Anfechtung muss in den Fällen der §§ 119, 120 ohne schuldhaftes Zögern (unverzüglich) erfolgen, nachdem der Anfechtungsberechtigte von dem Anfechtungsgrund Kenntnis erlangt hat. Die einem Abwesenden gegenüber erfolgte Anfechtung gilt als rechtzeitig erfolgt, wenn die Anfechtungserklärung unverzüglich abgesendet worden ist.

(2) Die Anfechtung ist ausgeschlossen, wenn seit der Abgabe der Willenserklärung zehn Jahre verstrichen sind.»

[6] Nel BGB la nozione di «unverzüglich» è quindi definita come «ohne schuldhaftes Zögern», ossia «senza indugio colpevole» (o «senza indugiare colpevolmente»). L'indugio, inteso come esitazione o ritardo, non è colpevole se le circostanze non permettono un'attuazione immediata a causa, per esempio, della necessità di compiere previamente determinati atti, per ricorrere a una consulenza legale oppure perché è concesso un periodo di riflessione, ovvero se vi sono motivi oggettivi per non agire subito. Se non vi sono simili motivi, l'atto va compiuto immediatamente («sofort», p. es. § $271 \mathrm{BGB}$ ).

[7] Questa distinzione tra «unverzüglich»e «sofort» è confermata per la legislazione federale svizzera da SNOzzI (2015, s. v. «indugio»): «senza indugio: sollecitamente, senza indebiti ritardi o dilazioni». I traducenti forniti sono, rispettivamente, «unverzüglich» e «sans délai». Il termine «indebito» corrisponde a «nicht schuldhaft» del BGB.

[8] Solo motivi oggettivi, quindi, possono dilazionare l'esecuzione di un atto di cui la legge prescrive un'attuazione «senza indugio». In assenza di motivi oggettivi, «senza indugio» equivale a «immediatamente». Infatti, il legislatore usa «senza indugio» quando l'esistenza di motivi oggettivi è certa oppure può essere presunta in base alla prassi o alla consuetudine, ma tali motivi non sussistono necessariamente in ogni situazione analoga.

[9] Per illustrare fino a che punto possa spingersi la complessità della problematica, si può considerare un esempio, tratto dalla legislazione ticinese, che testimonia una notevole compresenza, entro uno spazio ridotto, di diverse indicazioni relative alla tempistica prescritta. Ĺarticolo 11 capoversi 3 e 4 della legge del 22 gennaio 2018 sull'esercizio della prostituzione (Raccolta delle leggi del Cantone Ticino, n. 550.500) recita:

$\ll^{3}$ Il gerente [di un locale erotico] è tenuto a segnalare senza indugio alle competenti autorità situazioni che potrebbero originare inchieste penali. 
${ }^{4}$ In caso di assenza o di impedimento temporaneo, il gerente deve essere immediatamente sostituito da un supplente in grado di assumersi i compiti demandati dalla presente legge e dal regolamento di applicazione. Tale persona deve essere celermente notificata al Dipartimento ed essere in possesso dei requisiti dell'art. 10.»

[10] Lo stesso soggetto è chiamato a compiere tre atti con tempistiche diverse o diversamente connotate. Se per la segnalazione di cui al capoverso 3 il gerente può eventualmente attendere («senza indugio») fino a quando disponga di elementi concreti per ipotizzare l'esistenza delle situazioni in questione e se la sostituzione del gerente deve avvenire subito («immediatamente», cpv. 4), in modo che non si produca la pur minima vacanza nella gestione del locale, non è chiaro in quale intervallo temporale si collochi la «celerità» (cpv. 4) con cui il gerente deve notificare il proprio sostituto al Dipartimento: deve farlo subito o gli è concesso, se giustificato da motivi oggettivi, un certo lasso di tempo da determinare caso per caso?

[11] La problematica è dunque complessa e talvolta intricata. Il presente contributo può essere soltanto un primo approccio, necessariamente incompleto, per cercare di mettere a fuoco la materia nel contesto della legislazione federale.

\section{L'imperativo di celerità tra Costituzione federale e procedura penale}

[12] In ambito penale l'esigenza di agire senza tardare è espressa attraverso il cosiddetto imperativo di celerità («Beschleunigungsgebot», «principe de célérité») sancito nell'articolo 5 capoverso 1 del Codice di procedura penale (CPP; RS 312.0):

$\ll^{1}$ Le autorità penali avviano senza indugio i procedimenti penali e li portano a termine senza ritardi ingiustificati.

1 Die Strafbehörden nehmen die Strafverfahren unverzüglich an die Hand und bringen sie ohne unbegründete Verzögerung zum Abschluss.

${ }^{1}$ Les autorités pénales engagent les procédures pénales sans délai et les mènent à terme sans retard injustifié.»

[13] Notiamo che la terminologia usata è quella canonica, con le tre espressioni che indicano una celerità subordinata alle circostanze specifiche: «senza indugio», «unverzüglich» e «sans délai».

[14] L'imperativo di celerità è già presente nell'articolo 29 capoverso 1 della Costituzione federale (Cost.; RS 101), dove si fa garante di un giudizio reso in tempi adeguati:

« ${ }^{1}$ In procedimenti dinanzi ad autorità giudiziarie o amministrative, ognuno ha diritto alla parità ed equità di trattamento, nonché ad essere giudicato entro un termine ragionevole.

${ }^{1}$ Jede Person hat in Verfahren vor Gerichts- und Verwaltungsinstanzen Anspruch auf gleiche und gerechte Behandlung sowie auf Beurteilung innert angemessener Frist. 
${ }^{1}$ Toute personne a droit, dans une procédure judiciaire ou administrative, à ce que sa cause soit traitée équitablement et jugée dans un délai raisonnable.»

[15] Ed è ribadito nell'articolo 31 capoversi 2 e 3 Cost.:

« $^{2}$ Chi è privato della libertà ha diritto di essere informato immediatamente, in una lingua a lui comprensibile, sui motivi di tale privazione e sui diritti che gli spettano. $[\ldots]$

${ }^{3}$ Chi viene incarcerato a titolo preventivo ha diritto di essere prontamente tradotto davanti al giudice. [...]

${ }^{2}$ Jede Person, der die Freiheit entzogen wird, hat Anspruch darauf, unverzüglich und in einer ihr verständlichen Sprache über die Gründe des Freiheitsentzugs und über ihre Rechte unterrichtet zu werden. [...]

${ }^{3}$ Jede Person, die in Untersuchungshaft genommen wird, hat Anspruch darauf, unverzüglich einer Richterin oder einem Richter vorgeführt zu werden; [...]

2 Toute personne qui se voit privée de sa liberté a le droit d'être aussitôt informée, dans une langue qu'elle comprend, des raisons de cette privation et des droits qui sont les siens. [...]

${ }^{3}$ Toute personne qui est mise en détention préventive a le droit d'être aussitôt traduite devant un ou une juge, qui prononce le maintien de la détention ou la libération. [...]»

[16] L'immediatezza (cpv. 2) e la prontezza (cpv. 3) con cui vanno eseguiti gli atti nelle versioni italiana e francese risultano relativizzate nella versione tedesca, dove l'esecuzione sembra ammettere una dilazione («unverzüglich»). L'imperativo di celerità conosce dunque, nel CPP, una precisazione essenziale rispetto alla Costituzione attraverso l'uso di «senza indugio» (art. 5 $\mathrm{CPP}$ ) al posto di «immediatamente» e «prontamente» (art. 31 cpv. 2 e 3 Cost.). Sulla tempistica di questi due atti e le relative condizioni che li determinano torneremo più avanti trattando le disposizioni corrispettive della loro attuazione nel CPP (art. 209 cpv. 3 e 219 cpv. 1).

\section{Immediatezza e indugio nella legislazione federale}

[17] Le espressioni quali «senza indugio», «immediatamente» e «subito» rientrano nel campo delle norme (o nozioni) giuridiche indeterminate, che secondo Scolari $(2002,137$, n. 396) «esprimono un concetto astratto che deve essere precisato in ogni specifica situazione e applicato ad uno stato di fatto concreto. Sono perciò suscettibili di più determinazioni, a seconda del caso, del luogo e del tempo». Come detto, «immediatamente» e «subito» non lasciano di massima alcun margine temporale, mentre «senza indugio» ammette se del caso il trascorrere di un certo tempo: occorre valutare di volta in volta quale sia l'espressione opportuna.

[18] Per indagare l'applicazione di questa regola nella legislazione federale ci serviremo di esempi concreti. 


\section{1. «unverzüglich»}

[19] Se un atto va compiuto senza tardare, ossia se va compiuto non appena non vi sono (più) eventuali motivi oggettivi che possano differirlo, nella legislazione federale si usano i termini «unverzüglich», «senza indugio» e «sans délai». A volte sono utilizzate anche le varianti sinonimiche «ohne Verzug»e «sans retard», più raramente «sans tarder».

\subsubsection{Esempi conformi}

[20] art. 39 cpv. 2 CPP:

$~^{5}$ Erscheinen mehrere Strafbehörden als örtlich zuständig, so informieren sich die beteiligten Staatsanwaltschaften unverzüglich über die wesentlichen Elemente des Falles und bemühen sich um eine möglichst rasche Einigung.

${ }^{5}$ Se più autorità penali risultano competenti per territorio, i pubblici ministeri interessati si comunicano senza indugio gli elementi essenziali del caso e si adoperano per raggiungere un'intesa il più rapidamente possibile.

${ }^{5}$ Lorsque plusieurs autorités paraissent compétentes à raison du lieu, les ministères publics concernés se communiquent sans délai les éléments essentiels de l'affaire et s'entendent aussi vite que possible sur le for.»

[21] La comunicazione non deve essere istantanea ma va comunque fatta non appena possibile.

[22] art. 205 cpv. 2 CPP:

$«^{2}$ Wer verhindert ist, einer Vorladung Folge zu leisten, hat dies der vorladenden Behörde unverzüglich mitzuteilen; er oder sie hat die Verhinderung zu begründen und soweit möglich zu belegen.

${ }^{2}$ Chi è impedito di dar seguito a una citazione deve comunicarlo senza indugio all'autorità citante; l'impedimento va motivato e per quanto possibile provato.

${ }^{2}$ Celui qui est empêché de donner suite à un mandat de comparution doit en informer sans délai l'autorité qui l'a décerné; il doit lui indiquer les motifs de son empêchement et lui présenter les pièces justificatives éventuelles.»

[23] Una persona citata non deve attendere troppo prima di comunicare all'autorità di avere un impedimento. Se l'impedimento è per esempio solo supposto e quindi la persona giustamente non comunica subito di averlo, lo farà se del caso quando ne avrà la certezza.

[24] La legge federale del 20 dicembre 1968 sulla procedura amministrativa (RS 172.021) usa il sinonimo «ohne Verzug»:

[25] art. 8 cpv. 1 e 2 :

«1 Die Behörde, die sich als unzuständig erachtet, überweist die Sache ohne Verzug der zuständigen Behörde. 
2 Erachtet die Behörde ihre Zuständigkeit als zweifelhaft, so pflegt sie darüber ohne Verzug einen Meinungsaustausch mit der Behörde, deren Zuständigkeit in Frage kommt.

${ }^{1}$ L'autorità che si reputa incompetente trasmette senz'indugio la causa a quella competente.

${ }^{2}$ L'autorità che dubita di essere competente provoca senza indugio uno scambio d'opinioni con quella che potrebbe esserlo.

${ }^{1}$ L'autorité qui se tient pour incompétente transmet sans délai l'affaire à l'autorité compétente.

${ }^{2}$ L'autorité qui tient sa compétence pour douteuse ouvre sans délai un échange de vues avec l'autorité qu'elle considère comme compétente.»

[26] La tempistica della trasmissione della causa dipende, per esempio, dai preparativi necessari per attuarla (si noti nel cpv. 1 la variante grafica «senz'indugio», piuttosto rara nella legislazione federale) e lo scambio d'opinioni andrà avviato dopo i dovuti accertamenti.

[27] art. 15 cpv. 1 e 2 della legge del 23 giugno 2000 sugli avvocati (RS 935.61):

«1 Die kantonalen Gerichts- und Verwaltungsbehörden melden der Aufsichtsbehörde ihres Kantons unverzüglich das Fehlen persönlicher Voraussetzungen nach Artikel 8 sowie Vorfälle, welche die Berufsregeln verletzen könnten.

${ }^{2}$ Die eidgenössischen Gerichts- und Verwaltungsbehörden melden der Aufsichtsbehörde des Kantons, in dem eine Anwältin oder ein Anwalt eingetragen ist, unverzüglich das Fehlen persönlicher Voraussetzungen nach Artikel 8 sowie Vorfälle, welche die Berufsregeln verletzen könnten.

${ }^{1}$ Le autorità giudiziarie e amministrative cantonali comunicano senza indugio all'autorità di sorveglianza del loro Cantone l'inadempimento delle condizioni personali di cui all'articolo 8 , nonché i fatti che potrebbero costituire una violazione delle regole professionali.

${ }^{2}$ Le autorità giudiziarie e amministrative federali comunicano senza indugio all'autorità di sorveglianza del Cantone nel cui registro è iscritto l'avvocato l'inadempimento delle condizioni personali di cui all'articolo 8 , nonché i fatti che potrebbero costituire una violazione delle regole professionali.

${ }^{1}$ Les autorités judiciaires et administratives cantonales annoncent sans retard à l'autorité de surveillance de leur canton le défaut d'une condition personnelle au sens de l'art. 8, ou les faits susceptibles de constituer une violation des règles professionnelles. ${ }^{2}$ Les autorités judiciaires et administratives fédérales annoncent sans retard à l'autorité de surveillance du canton au registre duquel l'avocat est inscrit le défaut d'une condition personnelle au sens de l'art. 8, ou les faits susceptibles de constituer une violation des règles professionnelles.» 
[28] Notiamo che la tempistica prescritta alle autorità cantonali e federali è la stessa e che, in virtù della locuzione avverbiale opportuna, ammette il differimento della comunicazione per il tempo necessario.

[29] Tuttavia non vi è sempre corrispondenza nelle tre versioni linguistiche. In molti casi un atto che va compiuto «unverzüglich», e quindi con la possibilità di differirlo se necessario, ha nelle altre lingue il suo corrispettivo in un atto che va eseguito «immediatamente» e «immédiatement», ovvero senza che vi sia tale possibilità. In altri casi è proprio l'avverbio (o la locuzione avverbiale), benché presenti corrispondenza nelle tre versioni linguistiche, a non essere adeguato alla situazione specifica.

\subsubsection{Esempi non conformi}

[30] art. 185 cpv. 4 Cost.:

« ${ }^{4}$ In dringlichen Fällen kann er Truppen aufbieten. Bietet er mehr als 4000 Angehörige der Armee für den Aktivdienst auf oder dauert dieser Einsatz voraussichtlich länger als drei Wochen, so ist unverzüglich die Bundesversammlung einzuberufen.

${ }^{4}$ In casi urgenti, può mobilitare truppe. Se mobilita in servizio attivo più di 4000 militari o se questa mobilitazione si estende presumibilmente oltre le tre settimane, convoca immediatamente l'Assemblea federale.

${ }^{4}$ Dans les cas d'urgence, il peut lever des troupes. S'il met sur pied plus de 4000 militaires pour le service actif ou que cet engagement doive durer plus de trois semaines, l’Assemblée fédérale doit être convoquée sans délai.»

[31] L'urgenza della situazione sembrerebbe imporre un'azione immediata. La versione italiana risulterebbe quindi corretta dal punto di vista dell'uso dell'avverbio che indica la tempistica: l'Assemblea federale va convocata immediatamente, cosa che le versioni tedesca e francese non riflettono. Notiamo peraltro che la versione italiana è esplicita, e quindi più precisa, nell'indicare chi convoca l'Assemblea federale, ossia il Governo (cfr. messaggio del Consiglio federale del 20 novembre 1996, FF 1997 I 376), mentre la formulazione impersonale del tedesco e del francese potrebbe suscitare dubbi in quanto la competenza di convocare l'Assemblea federale spetta normalmente al legislatore (cfr. art. 2 cpv. 3 e 33 cpv. 2 e 3 Cost.).

[32] art. 54 cpv. 5 della legge federale del 19 dicembre 1958 sulla circolazione stradale (LCStr; RS 741.01):

$«^{5}$ Von der Polizei abgenommene Ausweise sind sofort der Entzugsbehörde zu übermitteln; diese entscheidet unverzüglich über den Entzug. Bis zu ihrem Entscheid hat die Abnahme eines Ausweises durch die Polizei die Wirkung des Entzugs.

${ }^{5}$ Le licenze sequestrate dalla polizia sono subito trasmesse all'autorità incaricata di revocarle, la quale prende immediatamente una decisione. Fino al momento della decisione, il sequestro da parte della polizia ha l'effetto di una revoca. 
${ }^{5}$ Les permis saisis par la police sont immédiatement transmis à l'autorité compétente, qui se prononce sans délai sur le retrait. Jusqu'à décision de l'autorité, la saisie opérée par la police a les mêmes effets qu'un retrait du permis.»

[33] In questo esempio si vede bene la distinzione concettuale tra «unverzüglich/senza indugio/sans délai» e «sofort/immediatamente/immédiatement». La polizia può e deve trasmettere subito le licenze, mentre l'autorità difficilmente potrà decidere subito. Essa ha quindi a disposizione il tempo necessario per farlo. E infatti, nel secondo periodo, si dà per scontato che «fino al momento della decisione» intercorra un certo tempo. Se le versioni tedesca e francese riflettono questa situazione, nella versione italiana l'avverbio «immediatamente» è da ritenersi impreciso e sarebbe stato opportuno usare «senza indugio».

[34] art. 64 cpv. 2 e 4 della legge federale del 16 dicembre 2005 sugli stranieri e la loro integrazione (LStrI; RS 142.20):

$«^{2}$ Verfügen die Ausländerinnen und Ausländer, die sich illegal in der Schweiz aufhalten, über einen gültigen Aufenthaltstitel eines anderen Staates, der durch eines der Schengen-Assoziierungsabkommen gebunden ist (Schengen-Staat), so sind sie formlos aufzufordern, sich unverzüglich in diesen Staat zu begeben. [...].

${ }^{4}$ Die zuständigen kantonalen Behörden bestimmen für unbegleitete minderjährige Ausländerinnen und Ausländer unverzüglich eine Vertrauensperson, die deren Interessen während des Wegweisungsverfahrens wahrnimmt.

${ }^{2}$ Lo straniero che soggiorna illegalmente in Svizzera e che è in possesso di un titolo di soggiorno valido rilasciato da un altro Stato vincolato da un accordo di associazione alla normativa di Schengen (Stato Schengen) è invitato senza formalità a recarsi immediatamente in tale Stato. [...].

${ }^{4}$ Le competenti autorità cantonali nominano senza indugio una persona di fiducia che difenda durante la procedura di allontanamento gli interessi dello straniero minorenne non accompagnato.

${ }^{2}$ L'étranger qui séjourne illégalement en Suisse et qui dispose d'un titre de séjour valable délivré par un autre État lié par l'un des accords d'association à Schengen (État Schengen) est invité sans décision formelle à se rendre immédiatement dans cet État. [...].

${ }^{4}$ Les autorités cantonales compétentes désignent immédiatement une personne de confiance chargée de représenter, au cours de la procédure de renvoi, les intérêts de l'étranger mineur non accompagné.»

[35] Il rientro dello straniero secondo il capoverso 1 difficilmente potrà avvenire istantaneamente, motivo per cui la versione tedesca prescrive che la partenza può avvenire con un eventuale ritardo giustificato («unverzüglich»), cosa che le altre due versioni linguistiche, utilizzando gli avverbi «immediatamente»e «immédiatement», non rispecchiano. Nel capoverso 4, invece, la versione italiana («senza indugio») è in linea con quella tedesca («unverzüglich») nel concedere il tempo necessario alla nomina di una persona di fiducia, contrariamente alla versione francese («immédiatement»). 
[36] art. 65 cpv. 1 LstrI:

"1 Wird die Einreise bei der Grenzkontrolle am Flughafen verweigert, so hat die Ausländerin oder der Ausländer die Schweiz unverzüglich zu verlassen.

${ }^{1}$ Se l'entrata in Svizzera è rifiutata al momento del controllo di confine all'aeroporto, lo straniero deve lasciare immediatamente la Svizzera.

${ }^{1}$ Si l'entrée en Suisse est refusée à un étranger lors du contrôle à la frontière à l'aéroport, il est tenu de quitter sans délai le territoire suisse.»

[37] In questo caso, nelle versioni tedesca («unverzüglich») e francese («sans délai») per l'abbandono del Paese è ammessa una dilazione eventualmente necessaria, mentre quella italiana prevede un'esecuzione istantanea.

[38] art. $111 i$ cpv. 8 LstrI:

$«^{8}[\ldots]$ Das SEM ersucht die Zentraleinheit unverzüglich um vorzeitige Vernichtung dieser Daten, sobald es Kenntnis davon erhält, dass die Ausländerin oder der Ausländer: $[\ldots]$.

${ }^{8}[\ldots]$ La SEM chiede senza indugio all'unità centrale di distruggere anzitempo tali dati non appena viene a conoscenza del fatto che lo straniero: $[\ldots]$.

${ }^{8}[\ldots]$ Le SEM demande à l'unité centrale de procéder à la destruction anticipée de ces données dès qu'il a connaissance du fait que l'étranger: [...].»

[39] In questo esempio salta all'occhio l'assenza, nella versione francese, dell'avverbio che indica la tempistica. Le versioni italiana («senza indugio») e tedesca («unverzüglich») sembrano esprimere la possibilità di dilazionare la richiesta di distruggere i dati, ma in realtà la Segreteria di Stato deve farlo «non appena viene a conoscenza» di determinati fatti, e quindi deve agire immediatamente. Sarebbe superfluo indicare l'avverbio poiché l'immediatezza è già espressa. La versione francese, con l'omissione dell'avverbio, risulta quindi essere quella conforme.

[40] art. 5 cpv. 3 dell'ordinanza del 23 marzo 2005 concernente la salvaguardia della sovranità sullo spazio aereo (RS 748.111.1):

« $^{3}$ Sie [die Luftwaffe] meldet Luftfahrzeuge, von denen sie feststellt, dass sie die Lufthoheit verletzen oder die Luftverkehrsregeln in schwer wiegender Weise verletzen, unverzüglich den Organen der Flugsicherung.

${ }^{3}$ Esse [le Forze aeree] annunciano senza indugio agli organi della sicurezza aerea gli aeromobili da parte dei quali constatano una violazione dello spazio aereo o una violazione grave delle norme sulla navigazione aerea.

${ }^{3}$ Elles [les Forces aériennes] annoncent sans délai aux services de la navigation aérienne les aéronefs dont elles constatent qu'ils violent la souveraineté sur l'espace aérien ou enfreignent gravement les règles de l'air.» 
[41] Una volta accertato che un aeromobile ha violato lo spazio aereo o le norme pertinenti, le Forze aeree non saranno presumibilmente legittimate a differire l'annuncio agli organi competenti, cosa che invece lascia pensare l'uso di «unverzüglich», «senza indugio» e «sans délai». Verosimilmente la situazione richiederebbe piuttosto che il fatto sia annunciato «immediatamente» («sofort», «immédiatement»). In caso di violazione, secondo l'articolo 15 capoverso 1 le Forze aeree e gli organi competenti riferiscono all'Ufficio federale dell'aviazione civile (UFAC), il quale informa a sua volta le autorità superiori se la violazione è grave:

[42] art. 15 cpv. 2:

$«^{2}$ In schwer wiegenden Fällen unterrichtet das BAZL unverzüglich das UVEK zu handen des Bundesrates sowie gegebenenfalls die Direktion für Völkerrecht.

${ }^{2}$ In casi gravi, l'UFAC informa immediatamente il DATEC, all'attenzione del Consiglio federale, e, se necessario, la Direzione del diritto internazionale pubblico.

${ }^{2}$ Dans les cas de violation grave, l'OFAC avise immédiatement le DETEC, qui en informe le Conseil fédéral et, le cas échéant, la DDIP.»

[43] L'informazione dell'UFAC dovrà probabilmente pure essere istantanea, cosa che esprimono le versioni italiana («immediatamente») e francese («immédiatement») ma non quella tedesca («unverzüglich»), che sembra ammettere un differimento.

\section{2. «sofort»}

[44] Notiamo che di norma «sofort» è reso con «immediatamente» e «immédiatement», mai (da quanto è stato possibile verificare) con «senza indugio» e «sans délai». Alcuni esempi:

[45] art. 165 cpv. 1 Cost.:

«1 Ein Bundesgesetz, dessen Inkrafttreten keinen Aufschub duldet, kann von der Mehrheit der Mitglieder jedes Rates dringlich erklärt und sofort in Kraft gesetzt werden. $[\ldots]$.

${ }^{1}$ Le leggi federali la cui entrata in vigore non possa essere ritardata possono essere dichiarate urgenti ed essere messe immediatamente in vigore dalla maggioranza dei membri di ciascuna Camera. [...].

${ }^{1}$ Une loi fédérale dont l'entrée en vigueur ne souffre aucun retard peut être déclarée urgente et entrer immédiatement en vigueur par une décision prise à la majorité des membres de chacun des conseils. [... ].»

[46] Per loro natura le leggi dichiarate urgenti entrano in vigore subito, e quindi sarebbe concettualmente impossibile prescrivere che debbano entrare in vigore «senza indugio». 
[47] art. 119 cpv. 2 LstrI:

$«^{2}$ Von der Strafverfolgung, der Überweisung an das Gericht oder der Bestrafung kann abgesehen werden, wenn die betroffene Person:

a. sofort ausgeschafft werden kann;

${ }^{2}$ Si può prescindere dal procedimento penale, dal rinvio a giudizio o dalla punizione se l'autore:

a. può essere immediatamente allontanato od espulso;

${ }^{2}$ Le juge peut renoncer à poursuivre l'étranger, à le renvoyer devant le tribunal ou à lui infliger une peine:

a. si le renvoi ou l'expulsion peut être exécuté immédiatement;»

[48] Il procedimento penale conosce un'importante svolta se è possibile eseguire subito l'allontanamento o l'espulsione.

[49] Nella LCStr assistiamo a un'alternanza di espressioni nelle versioni italiana e francese:

art. 27 cpv. 2: «sofort/immediatamente/immédiatement»

art. 51 cpv. 1: «sofort/subito/immédiatement»

art. 51 cpv. 3: «sofort/immediatamente/tout de suite»

art. 54 cpv. 5: «sofort/subito/immédiatement»

[50] Dal momento che la dovuta istantaneità è espressa con termini sinonimici, e che non abbiamo casi di «senza indugio/sans délai», il contenuto normativo resta impregiudicato.

[51] Nell'ambito della legge del 18 marzo 2016 sulle multe disciplinari (RS 314.1) regna un'immediatezza, temporale ma anche spaziale, che implica il pagamento delle multe istantaneamente e sul posto (resa in ogni caso con «immediatamente» e «immédiatement»):

[52] art. 6 cpv. 1-3:

" ${ }^{1}$ Wird die beschuldigte Person anlässlich der Widerhandlung identifiziert, so kann sie die Busse sofort oder innerhalb von 30 Tagen (Bedenkfrist) bezahlen.

2 Bezahlt sie sofort, so wird eine Quittung ohne ihren Namen ausgestellt.

${ }^{3}$ Bezahlt sie nicht sofort, so muss sie ihre Personalien angeben und erhält ein Bedenkfristformular sowie einen Einzahlungsschein. [...].

${ }^{1}$ Se è identificato al momento dell'infrazione, l'imputato può pagare la multa immediatamente o entro 30 giorni (termine di riflessione).

${ }^{2}$ Se paga immediatamente la multa, gli viene rilasciata una ricevuta che non menziona il suo nome.

${ }^{3}$ Se non paga immediatamente la multa, deve fornire i suoi dati personali e riceve un modulo concernente il termine di riflessione e una cedola di versamento. [...]. 
${ }^{1}$ Si le prévenu est identifié lors de l'infraction, il peut payer l'amende immédiatement ou dans un délai de 30 jours (délai de réflexion).

${ }^{2}$ S'il paie l'amende immédiatement, il reçoit une quittance ne mentionnant pas son nom.

${ }^{3}$ S'il ne paie pas l'amende immédiatement, il doit justifier de son identité et reçoit un formulaire prévoyant un délai de réflexion et un bulletin de versement. [... .»

[53] Il significato di «sofort» riferito a un atto che va compiuto immediatamente e senza tener conto di eventuali motivi che potrebbero differirlo emerge in modo chiaro nell'ambito del Codice di procedura civile (CPC; RS 272), dove l'avverbio esprime un'immediatezza contestuale alla contingenza processuale. Lo mostrano questi esempi:

[54] art. 237 cpv. 1:

«1 Das Gericht kann einen Zwischenentscheid treffen, wenn durch abweichende oberinstanzliche Beurteilung sofort ein Endentscheid herbeigeführt und so ein bedeutender Zeit- oder Kostenaufwand gespart werden kann.

${ }^{1}$ Il giudice può emanare una decisione incidentale quando un diverso giudizio dell'autorità giudiziaria superiore potrebbe portare immediatamente all'emanazione di una decisione finale e con ciò si potrebbe conseguire un importante risparmio di tempo o di spese.

${ }^{1}$ Le tribunal peut rendre une décision incidente lorsque l'instance de recours pourrait prendre une décision contraire qui mettrait fin au procès et permettrait de réaliser une économie de temps ou de frais appréciable.»

[55] Si noti che il testo francese rinuncia all'avverbio. Non si può comunque escludere che «sofort» sia in realtà da intendere come «unmittelbar» e quindi non nell'accezione temporale ma piuttosto in quella processuale (senza necessità di altri passi procedurali), che corrisponde pure a «immediatamente». Proprio in ambito processuale, infatti, «immediatamente» può riferirsi ad atti che non necessitano di ulteriori fasi e che possono essere disposti direttamente.

[56] art. 257 cpv. 1 lett. a:

«1 Das Gericht gewährt Rechtsschutz im summarischen Verfahren, wenn:

a. der Sachverhalt unbestritten oder sofort beweisbar ist; [...]

${ }^{1}$ Il giudice accorda tutela giurisdizionale in procedura sommaria se:

a. i fatti sono incontestati o immediatamente comprovabili; [...]

${ }^{1}$ Le tribunal admet l'application de la procédure sommaire lorsque les conditions suivantes sont remplies:

a. l'état de fait n'est pas litigieux ou est susceptible d'être immédiatement prouvé;» 
[57] art. 373 cpv. 6:

« ${ }^{6}$ Verstösse gegen die Verfahrensregeln sind sofort zu rügen, andernfalls können sie später nicht mehr geltend gemacht werden.

${ }^{6}$ Le violazioni di regole di procedura devono essere eccepite immediatamente, pena la perenzione.

6 Toute violation des règles de procédure doit être immédiatement invoquée; à défaut, elle ne peut l'être par la suite.»

[58] Nel CPP «sofort» è sempre reso con «immediatamente»e «immédiatement», specialmente in relazione alla distruzione di dati e all'immediatezza dell'esecuzione di atti processuali. In due casi, tuttavia, l'italiano e il francese si scostano:

[59] art. 365 cpv. 2:

$«^{2}$ Es erlässt seinen Entscheid schriftlich und begründet ihn kurz. Hat eine Verhandlung stattgefunden, so eröffnet es seinen Entscheid sofort mündlich.

2 Il giudice pronuncia la decisione per scritto e la motiva succintamente. Se si è svolto un dibattimento, la decisione è comunicata oralmente seduta stante.»

[60] Al di là della precisione plastica del termine usato, ci si può chiedere se la scelta di «seduta stante» al posto di «immediatamente» non sia (pure) dovuta all'esigenza di evitare una cacofonia risultante dalla concomitanza con l'avverbio «oralmente».

[61] art. 366 cpv. 3:

${ }^{3}$ Hat sich die beschuldigte Person selber in den Zustand der Verhandlungsunfähigkeit versetzt oder weigert sie sich, aus der Haft zur Hauptverhandlung vorgeführt zu werden, so kann das Gericht sofort ein Abwesenheitsverfahren durchführen.

${ }^{3}$ Si le prévenu s'est lui-même mis dans l'incapacité de participer aux débats ou s'il refuse d'être amené de l'établissement de détention aux débats, le tribunal peut engager aussitôt la procédure par défaut.»

[62] Se l'avverbio «aussitôt» può essere ritenuto sinonimo di «immédiatement», è comunque notevole che le sole due occorrenze in tutto il Codice si presentino in vesti diverse: nel presente caso corrisponde a «sofort» e «immediatamente», mentre nell'altro (art. $131 \mathrm{cpv}$. 1) ha come corrispettivi «unverzüglich» e «senza indugio».

\section{3. «umgehend»}

[63] L'avverbio «umgehend», più vicino al significato di «sofort» che a quello di «unverzüglich», ha corrispondenze variabili nelle altre due lingue ufficiali. Nella versione italiana si alternano, con frequenze paragonabili, «immediatamente» e «senza indugio», ma non sembrano esservi 
occorrenze di «subito». Nella versione francese le espressioni corrispondenti sono «immédiatement», che pare prevalere, «sans délai» e «sans tarder». Alcuni esempi:

[64] art. 76 cpv. 4 LstrI:

« Die für den Vollzug der Wegweisung, der Ausweisung oder der Landesverweisung nach Artikel $66 a$ oder $66 a^{\text {bis }}$ StGB oder Artikel $49 a$ oder $49 a{ }^{\text {bis }}$ MStG notwendigen Vorkehren sind umgehend zu treffen.

${ }^{4}$ I passi necessari per l'esecuzione dell'allontanamento o dell'espulsione secondo la presente legge oppure per l'esecuzione dell'espulsione ai sensi dell'articolo 66 a o 66

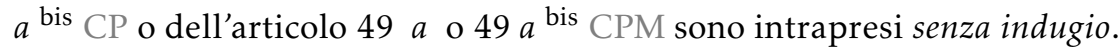

${ }^{4}$ Les démarches nécessaires à l'exécution du renvoi, de l'expulsion au sens de la présente loi ou de l'expulsion au sens des art. 66 a ou $66 a^{\text {bis }}$ CP ou $49 a$ ou $49 a^{\text {bis }}$ CPM doivent être entreprises sans tarder.»

[65] art. 77 cpv. 3 LstrI:

« $^{3}$ Die für den Vollzug der Weg- oder Ausweisung notwendigen Vorkehrungen sind umgehend zu treffen.

${ }^{3}$ I passi necessari per l'esecuzione dell'allontanamento o dell'espulsione sono intrapresi senza indugio.

${ }^{3}$ Les démarches nécessaires à l'exécution du renvoi ou de l'expulsion doivent être entreprises sans tarder.»

[66] Vista l'urgenza procedurale esistente in questo ambito, sembra di poter affermare che «umgehend» vada inteso come «sofort» e connoti piuttosto un atto da compiere subito, e non subordinatamente a condizioni specifiche. Sarebbe quindi stato opportuno usare «immediatamente» e «immédiatement».

[67] Nella legge federale del 22 giugno 2001 sulla cooperazione con la Corte penale internazionale (RS 351.6) vi sono sette occorrenze di «umgehend», corrispondenti a tre di «senza indugio», tre di «immediatamente» e una di «subito», ma a sette di «immédiatement». Nella versione italiana si nota quindi una diversificazione che si contrappone alla compattezza terminologica delle altre due versioni linguistiche. Nei tre casi di «senza indugio» la versione italiana sembra esprimere meglio delle altre il senso di un atto che non va compiuto necessariamente nell'immediato ma può essere differito per il tempo necessario. Si tratta infatti di situazioni in cui l'Ufficio centrale notifica alla Corte una decisione (art. 10 cpv. 4), la informa di un arresto con sequestro conservativo comunicatogli a sua volta (art. $18 \mathrm{cpv}$. 4) e le trasmette un'istanza di liberazione condizionale, di grazia, di appello o di revisione (art. 56). Proprio nell'ultimo esempio l'opportunità di una trasmissione effettuata «senza indugio» è confermata dal fatto che l'istanza va trasmessa «unitamente a tutti i documenti utili», la cui raccolta o compilazione può richiedere un certo intervallo di tempo. 
[68] L'articolo 85 capoverso 1 dell'ordinanza dell'11 febbraio 2004 sulla circolazione stradale militare (RS 510.710) offre un interessante esempio di compresenza di «umgehend» e «sofort»:

«1 Bei schweren Unfällen mit Militärfahrzeugen ist zusätzlich zum Beizug der Personen und Stellen nach Artikel 80 und zu den Meldungen nach Artikel 83 eine erste Meldung sofort telefonisch an die Pikettstelle des VBS zu erstatten und mittels entsprechendem Formular umgehend zu bestätigen.

${ }^{1}$ In caso di incidenti gravi con veicoli militari, in via aggiuntiva al ricorso alle persone e agli organi secondo l'articolo 80 e alle notifiche di cui all'articolo 83, la prima notifica deve essere fatta subito per telefono al servizio di picchetto del DDPS e confermata senza indugio mediante l'apposito modulo.

${ }^{1}$ Lors d'accidents graves impliquant des véhicules militaires, une première annonce doit être faite immédiatement au service de piquet du DDPS puis confirmée sans délai au moyen du formulaire approprié, en sus du recours aux personnes et organes visés à l'art. 80 et aux déclarations visées à l'art. 83.»

[69] La differenziazione delle due tempistiche, espresse con due avverbi diversi, sembra giustificata dalla necessità di telefonare immediatamente ma di compilare e inviare il modulo non appena possibile. In questo caso «umgehend» equivale a «unverzüglich». I termini usati nelle altre due versioni linguistiche sono quindi adeguati, ma in quella francese la differenza tra i due atti da compiere risulta opaca in quanto si è omesso di menzionare la modalità della prima notifica («telefonisch», «per telefono»).

[70] Nelle sole tre occorrenze riscontrabili nel CPP «umgehend» è reso sempre con «immediatamente», rispettivamente una volta con «sans délai»e due con «immédiatement».

[71] art. 95 cpv. 2 CPP:

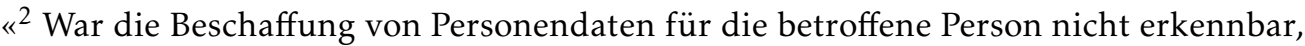
so ist diese umgehend darüber zu informieren. Die Information kann zum Schutze überwiegender öffentlicher oder privater Interessen unterlassen oder aufgeschoben werden.

${ }^{2}$ Se non poteva ravvisare di essere oggetto di una raccolta di dati, l'interessato deve esserne immediatamente informato. Si può rinunciare all'informazione o differirla per proteggere interessi pubblici o privati preponderanti.

${ }^{2}$ Si des données personnelles sont collectées à l'insu de la personne concernée, celleci doit en être informée sans délai. L'autorité peut renoncer à cette information ou l'ajourner si un intérêt public ou privé prépondérant l'exige.»

[72] La possibilità di differire l'informazione, data nel secondo periodo, fa capire che «umgehend» del primo periodo sia da intendere come «sofort». La versione italiana è quindi conforme, contrariamente a quella francese. 
[73] Diverso il caso dell'articolo 214 capoverso $1 \mathrm{CPP}$, dove tutte e tre le versioni linguistiche esprimono l'esigenza di un'azione istantanea:

" Wird eine Person vorläufig festgenommen oder in Untersuchungs- oder Sicherheitshaft gesetzt, so benachrichtigt die zuständige Strafbehörde umgehend:

${ }^{1}$ Se una persona è arrestata provvisoriamente o posta in carcerazione preventiva o di sicurezza, l'autorità penale competente ne avvisa immediatamente:

${ }^{1}$ Si une personne est arrêtée provisoirement ou mise en détention provisoire ou en détention pour des motifs de sûreté, l'autorité pénale compétente informe immédiatement:»

[74] Nell'articolo 307 capoverso $3 \mathrm{CPP}$, infine, la necessità di compiere subito un atto necessario al celere svolgimento di un procedimento penale trova riscontro in una rigorosa omogeneità dei termini impiegati nelle tre versioni linguistiche:

${ }^{3}$ Die Polizei hält ihre Feststellungen und die von ihr getroffenen Massnahmen laufend in schriftlichen Berichten fest und übermittelt diese nach Abschluss ihrer Ermittlungen zusammen mit den Anzeigen, Protokollen, weiteren Akten sowie sichergestellten Gegenständen und Vermögenswerten umgehend der Staatsanwaltschaft.

${ }^{3}$ La polizia annota di volta in volta in rapporti scritti gli accertamenti da essa compiuti e le misure da essa adottate e, dopo la conclusione delle indagini, li trasmette immediatamente al pubblico ministero unitamente alle denunce, ai verbali, agli altri atti nonché agli oggetti e ai valori patrimoniali messi al sicuro .

${ }^{3}$ La police établit régulièrement des rapports écrits sur les mesures qu'elle a prises et les constatations qu'elle a faites et les transmet immédiatement après ses investigations au ministère public avec les dénonciations, les procès-verbaux, les autres pièces, ainsi que les objets et les valeurs mis en sûreté.»

\section{Un caso particolare: «unverzüglich» nel CPP}

[75] Abbiamo già visto diversi esempi del CPP, nell'ambito del quale la problematica in esame è molto sentita in particolare a causa dell'imperativo di celerità di cui si è detto sopra. Vale però la pena guardare più da vicino alcuni casi di «unverzüglich», «senza indugio» e "sans délai» in questo Codice. A mo' di premessa va precisato che la stesura della versione italiana del Codice ha goduto di un'attenzione particolare proprio per alcuni aspetti specifici, tra cui la resa di «unverzüglich». Come evidenzia EGger $(2019,92)$, la coerenza terminologica è stata fortemente sorvegliata e voluta, tanto da risultare persino programmatica e sistematica:

«Nel Codice di procedura penale [...] si è prestata particolare attenzione a esprimere in modo sistematicamente uguale gli intervalli di tempo procedurali, distinguendo 
cioè rigorosamente il significato di indicazioni solitamente vaghe e sinonimiche come senza indugio (unverzüglich, sans délai), immediatamente (sofort, immédiatement), rapidamente (rasch, vite), un breve termine (eine kurze Nachfrist, un bref délai).»

[76] Nella panoramica sui lavori legati alla stesura e traduzione del CPP fornita da EGGER/GRANDI 2008 si insisteva già su questo specifico argomento: «Ad es. l'avverbio <unverzüglich» era stato tradotto in diversi modi «senza indugio, immediatamente, quanto prima`, non tutti equivalenti.» (nota 14).

\subsection{Diversificazione terminologica nel CPP}

[77] Alle 49 occorrenze di «unverzüglich» corrispondono, rispettivamente, 46 di «senza indugio» e tre di «immediatamente», nonché 14 di «sans délai», 11 di «sans retard», 23 di «immédiatement» e una di «aussitôt». Di fronte alla marcata aderenza della versione italiana a quella tedesca, i tre casi in cui l'italiano usa «immediatamente» (art. 209 cpv. 3, 217 cpv. 3 lett. b, 224 cpv. 2) meritano un approfondimento.

[78] art. 209 cpv. 3:

«3 Die Behörde informiert die vorgeführte Person unverzüglich und in einer ihr verständlichen Sprache über den Grund der Vorführung, nimmt die Verfahrenshandlung vor und entlässt sie danach unverzüglich, es sei denn, sie beantrage die Anordnung der Untersuchungs- oder der Sicherheitshaft.

${ }^{3}$ L'autorità informa senza indugio l'interessato, in una lingua a lui comprensibile, sul motivo dell'accompagnamento, compie l'atto procedurale e lo rilascia immediatamente, eccetto che ne proponga la carcerazione preventiva o di sicurezza.

${ }^{3}$ L'autorité informe la personne amenée, sans délai et dans une langue qu'elle comprend, du motif du mandat d'amener, exécute l'acte de procédure et la libère ensuite immédiatement à moins qu'elle ne propose d'ordonner la détention provisoire ou la détention pour des motifs de sûreté.»

[79] Nella prima occorrenza di «unverzüglich» l'autorità informa l'interessato sul motivo dell'accompagnamento non appena avrà eseguito altri atti preliminari rispetto all'atto procedurale (un interrogatorio, nella maggior parte dei casi), tra cui ad esempio informarlo su altre circostanze specifiche. Nella seconda occorrenza l'autorità rilascia l'interessato subito dopo l'atto procedurale. L'alternativa al rilascio immediato non è un rilascio effettuato non appena possibile («senza indugio») bensì il non rilascio perché se ne propone la carcerazione preventiva o di sicurezza. Per maggiore chiarezza il testo tedesco, analogamente alle versioni italiana e francese, avrebbe dovuto esprimere l'immediatezza dell'atto usando l'avverbio «sofort». L'uso di avverbi differenti evidenzia la diversa tempistica legata ai due atti: informare la persona subordinatamente al compimento di altri atti, da un lato, e rilasciarla subito, dall'altro. L'esempio analogo dell'articolo 219 capoverso $3 \mathrm{CPP}$ conferma l'opportunità di diversificare gli avverbi e quindi la tempistica: 
«3 Ergeben die Abklärungen, dass Haftgründe nicht oder nicht mehr bestehen, so lässt sie die festgenommene Person sofort frei. Bestätigen die Abklärungen den Tatverdacht und einen Haftgrund, so führt sie die Person unverzüglich der Staatsanwaltschaft zu.

${ }^{3}$ Se dagli accertamenti risulta che non sussistono o sono venuti meno motivi di carcerazione, l'arrestato è liberato immediatamente. Se gli accertamenti confermano gli indizi di reato e i motivi di carcerazione, la polizia traduce senza indugio l'arrestato dinanzi al pubblico ministero.

${ }^{3}$ S'il ressort des investigations qu'il n'y a pas ou plus de motifs de détention, la personne arrêtée est immédiatement libérée. Si les investigations confirment les soupçons ainsi qu'un motif de détention, la police amène la personne sans retard devant le ministère public.»

[80] La liberazione dell'arrestato deve essere immediata, mentre la sua traduzione richiede verosimilmente l'esecuzione preliminare di qualche atto procedurale, circostanze che tutte e tre le versioni linguistiche esprimono coerentemente con gli avverbi adeguati.

[81] art. 217 cpv. 3 lett. b:

«3 Sie kann eine Person, die sie bei der Begehung einer Übertretung auf frischer Tat ertappt oder unmittelbar nach Begehung einer solchen Tat angetroffen hat, vorläufig festnehmen und auf den Polizeiposten bringen, wenn:

b. die Person nicht in der Schweiz wohnt und nicht unverzüglich eine Sicherheit für die zu erwartende Busse leistet;

${ }^{3}$ La polizia può arrestare provvisoriamente e condurre al posto di polizia chi è colto in flagranza di contravvenzione o sorpreso immediatamente dopo aver commesso una contravvenzione se:

b. non abita in Svizzera e non fornisce immediatamente una garanzia per la multa prevedibile;

${ }^{3}$ Elle peut arrêter provisoirement et conduire au poste toute personne qu'elle a surprise en flagrant délit de contravention ou intercepte immédiatement après un tel acte si:

b. la personne n'habite pas en Suisse et ne fournit pas immédiatement des sûretés pour l'amende encourue;»

[82] La situazione concreta impone la fornitura istantanea della garanzia. Se non la fornisce sul momento, l'interessato può essere arrestato provvisoriamente e condotto al posto di polizia. Non vi è la possibilità di fornire la garanzia dopo aver compiuto altri atti. Di conseguenza le versioni italiana e francese sono conformi, mentre quella tedesca dovrebbe usare «sofort».

[83] art. 224 cpv. 2:

«2 Bestätigen sich der Tatverdacht und die Haftgründe, so beantragt die Staatsanwaltschaft dem Zwangsmassnahmengericht unverzüglich, spätestens aber innert 48 
Stunden seit der Festnahme, die Anordnung der Untersuchungshaft oder einer Ersatzmassnahme. [...]

${ }^{2}$ Se gli indizi di reato e i motivi di carcerazione si confermano, il pubblico ministero, immediatamente ma al più tardi 48 ore dopo l'arresto, propone al giudice dei provvedimenti coercitivi di ordinare la carcerazione preventiva o una misura sostitutiva. $[\ldots]$

${ }^{2}$ Si les soupçons et les motifs de détention sont confirmés, le ministère public propose au tribunal des mesures de contrainte, sans retard mais au plus tard dans les 48 heures à compter de l'arrestation, d'ordonner la détention provisoire ou une mesure de substitution. [... ]»

[84] Per l'atto da eseguire è impartito un termine ultimo. Ciò significa implicitamente che l'atto non deve (necessariamente) essere compiuto subito, ma che vi è a disposizione il lasso di tempo indicato. Le versioni tedesca e francese («unverzüglich», «sans retard») esprimono bene questa circostanza, mentre quella italiana sembra in un certo senso contraddirsi: sarebbe stato opportuno usare «senza indugio». Un esempio simile, l'articolo 226 capoverso 1, viene a corroborare tale ipotesi in quanto presenta una situazione paragonabile quanto alla tempistica e usa gli stessi termini:

«1 Das Zwangsmassnahmengericht entscheidet unverzüglich, spätestens aber innert 48 Stunden nach Eingang des Antrags.

${ }^{1}$ Il giudice dei provvedimenti coercitivi decide senza indugio, ma al più tardi entro 48 ore da quando ha ricevuto la proposta del pubblico ministero.

${ }^{1}$ Le tribunal des mesures de contrainte statue immédiatement, mais au plus tard dans les 48 heures suivant la réception de la demande.»

[85] La locuzione avverbiale "senza indugio» usata in questo caso esprime che sia il pubblico ministero (art. 224 cpv. 2) sia il giudice dei provvedimenti coercitivi (art. $226 \mathrm{cpv}$. 1) hanno a disposizione un certo margine temporale, esplicitato in entrambi i casi, prima di compiere l'atto prescritto. È interessante notare che nel secondo esempio la versione francese, che nel primo era conforme, cambia avverbio e crea la stessa contraddizione che abbiamo nella versione italiana del primo esempio.

[86] Possiamo dunque affermare che in due dei tre casi in esame (art. 209 cpv. 3 e 217 cpv. 3 lett. b) la versione italiana, come quella francese, si è scostata opportunamente dalla versione tedesca, mentre nel terzo caso (art. 224 cpv. 2) se ne è allontanata indebitamente, al pari della versione francese.

\subsection{La tempistica nei commentari al CPP}

[87] In alcuni casi i commentari al CPP, che sono deputati a spiegare e chiarire il testo di legge, in realtà se ne scostano proprio nel trattare le disposizioni in cui figurano le espressioni 
«unverzüglich», «senza indugio» e «sans délai», determinando sinonimie improprie. In altri casi il commento lascia invece dubbi sull'uso degli avverbi indicanti le tempistiche nella legge. Fra gli innumerevoli esempi ne consideriamo tre relativi a disposizioni contenenti l'avverbio «unverzüglich»:

[88] art. 41 cpv. 1 :

«1 Will eine Partei die Zuständigkeit der mit dem Strafverfahren befassten Behörde anfechten, so hat sie dieser unverzüglich die Überweisung des Falles an die zuständige Strafbehörde zu beantragen.

${ }^{1}$ La parte che intende contestare la competenza dell'autorità investita del procedimento penale deve chiedere senza indugio a quest'ultima di rimettere il caso all'autorità penale competente.

${ }^{1}$ Lorsqu'une partie entend contester la compétence de l'autorité en charge de la procédure pénale, elle doit immédiatement demander à cette dernière de transmettre l'affaire à l'autorité pénale compétente.»

[89] La versione francese si scosta dalle altre due. In BernAsConi et al. 2010 si legge: «La richiesta di trasferimento del procedimento ad un'altra autorità deve essere formulata «senza indugio», e meglio non appena la parte è venuta a conoscenza delle circostanze rilevanti su cui si fonda la richiesta di cambiamento di foro.» (99). La formulazione «e meglio non appena» non risulta del tutto chiara: si intende «detto meglio», «vale a dire»? Oppure «non appena» indica un momento più vicino rispetto a «senza indugio»? Donatsch et al. 2014 spiegano: «Zwar nennt das Gesetz in diesem Zusammenhang keine Frist; die Unzuständigkeit ist aber unverzüglich geltend zu machen. Dies bedeutet, dass die Partei aktiv zu werden hat, sobald sie mit genügender Sicherheit erkennen kann, wer das Verfahren führt und welche gerichtsstandsrelevanten Tatsachen vorliegen, welche die Zuständigkeit infrage stellen können.» (243). Dal canto suo RiкLIN 2014 aggiunge: «Unverzüglichkeit ist gefordert, weil die Hinnahme der nach Meinung der betreffenden Parteien unzuständigen Behörde während längerer Zeit als Anerkennung des Gerichtsstandes durch konkludentes Verhalten gewertet werden kann und weil auch der Grundsatz von Treu und Glauben (vgl. StPO Art. 43 Abs. 2 lit. a) Unverzüglichkeit gebietet.» (151). Per il francese Jeanneret et al. 2019, pur usando l'avverbio «immédiatement» come nella legge, confermano di fatto che le versioni italiana e tedesca, che con «senza indugio» e «unverzüglich» ammettono il trascorrere di un certo tempo, sono quelle corrette: «Elle [une partie] doit le faire <immédiatement), c'est-à-dire aussitôt qu'elle dispose des éléments propres à motiver sa détermination.» (187).

[90] art. 219 cpv. 1:

«1 Die Polizei stellt nach der Festnahme unverzüglich die Identität der festgenommenen Person fest, informiert diese in einer ihr verständlichen Sprache über die Gründe der Festnahme und klärt sie im Sinne von Artikel 158 über ihre Rechte auf. [... ].

${ }^{1}$ Dopo l'arresto la polizia accerta senza indugio l'identità dell'arrestato, lo informa in una lingua a lui comprensibile sui motivi dell'arresto e, ai sensi dell'articolo 158, lo rende attento ai suoi diritti. [...]. 
${ }^{1}$ La police établit immédiatement après l'arrestation l'identité de la personne arrêtée, l'informe dans une langue qu'elle comprend des motifs de son arrestation et la renseigne sur ses droits au sens de l'art. 158. [... ].»

[91] Il commento di Bernasconi et al. 2010 dichiara sinonimi «senza indugio» e «immediatamente»: «La persona arrestata deve essere informata immediatamente (senza indugio) circa i motivi dell'arresto, la procedura avviata contro di lui, le relative ipotesi di reato [... ].» (426). Tuttavia, dal seguito delle spiegazioni risulta chiaro che l'informazione non è richiesta nell'immediato: «È opportuno che tali comunicazioni $[\ldots]$ siano fornite il più presto possibile, quindi senza necessariamente attendere l'inizio dell'interrogatorio [... ].» (426). Per la versione francese JEANNERET et al. 2019 forniscono il seguente commento: «Les opérations d'identification doivent avoir lieux immédiatement. Cela n'empêche toutefois pas la police d'ordonner d'autres mesures, plus longues, en vue d'identifier le suspect [... ].» (1403). E ancora:

«Immédiatement après l'arrestation, la police informe la personne arrêtée dans une langue qu'elle comprend des motifs de son arrestation et la renseigne sur ses droits au sens de CPP 158 (CPP 219 I, $1^{\text {re }}$ phr.). Il s'agit ici d'une concrétisation en droit suisse de CEDH 5 II qui dispose que toute personne arrêtée doit être informée, dans le plus court délai et dans une langue qu'elle comprend, des raisons de son arrestation et de toute accusation portée contre elle. Le droit suisse est cependant plus strict que le droit conventionnel puisqu'il impose une information immédiates. Des circonstances particulières, telles que le grand nombre de personnes arrêtées ou la nécessité de recourir à un interprète pour une langue peu courante en Suisse, peuvent retarder cette communication immédiate.» (1403 seg.).

[92] Proprio dalle spiegazioni fornite nell'ultimo commento, che configurano le situazioni che possono costituire motivi oggettivi per ritardare il compimento di un atto, si deduce che nel testo francese si sarebbe dovuto mettere «sans délai», analogamente all'italiano e al tedesco.

[93] art. 241 cpv. 3:

«3 Ist Gefahr im Verzug, so kann die Polizei die Untersuchung der nicht einsehbaren Körperöffnungen und Körperhöhlen anordnen und ohne Befehl Durchsuchungen vornehmen; sie informiert darüber unverzüglich die zuständige Strafbehörde.

${ }^{3}$ Se vi è pericolo nel ritardo, la polizia può ordinare l'ispezione di orifizi e cavità corporei non visibili esternamente e può, senza mandato, eseguire perquisizioni; essa ne informa senza indugio le autorità penali competenti.

${ }^{3}$ Lorsqu'il y a péril en la demeure, la police peut ordonner l'examen des orifices et des cavités du corps qu'il est impossible d'examiner sans l'aide d'un instrument et effectuer des perquisitions sans mandat; le cas échéant, elle en informe sans délai l'autorité pénale compétente.»

[94] Bernasconi et al. 2010 commentano: «In questi casi [cioè nei casi in cui la polizia ha essa stessa ordinato l'ispezione ed eseguito perquisizioni], l'autorità competente (ministero pubblico) deve 
essere informata (successivamente all'adozione della misura) senza indugio.» (483). L'informazione può quindi avvenire dopo il tempo necessario all'adozione della misura. Secondo Donatsch et al. 2014, dalla tempistica prescritta si può dedurre che i motivi d'indugio non sono infiniti e che in particolare non possono essere giustificati da contingenze burocratiche: «Unverzüglich» kann in Anbetracht der in allen Kantonen bestehenden Pikettorganisation nur so verstanden werden, dass auch nachts informiert werden muss [... ].» (1377). JEANNEREt et al. 2019, infine, spiegano in modo eloquente come si debba intendere la tempistica del testo di legge: «[...] la police doit informer l'autorité pénale compétente [...] de la démarche qu'elle a effectuée sans mandat. Cette information devra être communiquée ‘sans délaì, c'est-à-dire au plus tard après que la situation de péril en la demeure a disparu.» (1546).

\subsection{Il difficile rapporto tra legge e messaggio: l'articolo 209 capoverso $2 \mathrm{CPP}$}

[95] Talvolta le spiegazioni fornite nel messaggio del Consiglio federale a sostegno di un disegno di legge non chiariscono veramente il significato concreto di una nozione giuridica indeterminata come «senza indugio» $\mathrm{o}$ «immediatamente». Oppure la spiegano dando come sinonimo uno degli avverbi che rivestono in realtà un significato diverso nel linguaggio giuridico. Un esempio tratto dal CPP mostra questo rapporto a volte complicato.

[96] art. 209 cpv. 2:

$«^{2}$ Sie [die Polizei] weist der vorzuführenden Person den Vorführungsbefehl vor und führt sie unverzüglich oder zu der im Vorführungsbefehl genannten Zeit der Behörde $\mathrm{zu}$.

${ }^{2}$ La polizia esibisce il mandato di accompagnamento all'interessato e traduce costui dinanzi all'autorità, senza indugio o all'ora indicata nel mandato.

${ }^{2}$ Elle [la police] présente le mandat d'amener à la personne visée et la conduit devant l'autorité immédiatement ou à l'heure indiquée sur le mandat.»

[97] Notiamo che la versione francese prescrive un'immediatezza che le versioni italiana e tedesca non esprimono. Il fatto stesso che l'alternativa alla traduzione senza indugio sia di eseguirla all'ora indicata nel mandato lascia intendere che l'interessato non va necessariamente tradotto all'istante dinanzi all'autorità ma che possono esservi motivi per differirne la traduzione. La spiegazione di «senza indugio» fornita nel messaggio italiano (FF 2006 989, in particolare 1124 seg.) fa però capire che l'espressione che andava usata nel testo di legge è «immediatamente»:

«L'obbligo di cui al capoverso 2 di tradurre senza indugio l'interessato dinanzi all'autorità sussiste fatta salva un'eventuale disposizione contraria da parte di questa stessa autorità. In quanto dirige la procedura preliminare, il pubblico ministero può ad esempio disporre che l'interessato sia prima tradotto in polizia per primi accertamenti. In mancanza di un ordine specifico, l'interessato è invece tradotto senza indugio dinanzi al pubblico ministero.» 
[98] La traduzione dell'interessato è quindi differita se l'autorità impartisce un ordine diverso. Se non esiste un simile ordine, la persona va tradotta immediatamente. L'indicazione dell'ora nel mandato sembrerebbe significare che l'autorità abbia dato un ordine alternativo e concesso il tempo necessario per eseguirlo.

[99] Il messaggio tedesco (BB1 2006 1085, in particolare 1221) recita:

«Die Pflicht zur sofortigen Vorführung an die Behörde nach Absatz 2 steht unter dem Vorbehalt anders lautender Anordnungen dieser Behörde. So kann die Staatsanwaltschaft in ihrer Funktion als Leiterin des Vorverfahrens anordnen, dass eine vorzuführende Person zunächst der Polizei für erste Ermittlungen zuzuweisen ist. Ohne eine solche Anordnung hat indes eine unverzügliche Überstellung an die Staatsanwaltschaft zu erfolgen.»

[100] L'avverbio «unverzüglich» è dato come sinonimo di «sofortig» nel primo periodo e ritorna come aggettivo nel terzo. Dalla spiegazione fornita appare chiaro che il senso è quello di «sofort». [101] Il commento contenuto nel messaggio francese (FF 2006 1057, in particolare 1202) mette la questione nei giusti termini:

«L'obligation de conduire la personne visée devant l'autorité au sens de l'al. 2 s'applique sous réserve d'un ordre contraire donné par cette autorité. Le ministère public, en sa qualité d'autorité dirigeant l'instruction, peut ainsi ordonner qu'une personne soit d'abord remise à la police pour de premières investigations. A défaut d'ordre de ce genre, la personne visée doit immédiatement être remise au ministère public.»

[102] La subordinazione della traduzione immediata all'esistenza di ordini alternativi dell'autorità, che ne determinano il differimento, è espressa correttamente nel terzo periodo con l'avverbio «immédiatement», corrispondente alla legge. Nel primo periodo l'avverbio è stato omesso ma è implicito nella clausola di rimando «au sens de l'al. 2» (il cpv. 2 recita infatti «immédiatement»). [103] Possiamo quindi affermare che nelle versioni italiana e tedesca sarebbe stato corretto usare, rispettivamente, «immediatamente» e «sofort», analogamente a quella francese. La traduzione dell'interessato deve avvenire immediatamente, salvo che l'autorità non abbia fissato un'ora specifica che permetta di eseguire in via preliminare altri ordini eventualmente impartiti.

\section{I difetti nascosti del Codice delle obbligazioni}

[104] Una nozione indeterminata come «immediatamente» non stabilisce i tempi concreti da rispettare per compiere un atto. Non è affatto scontato che l'atto in questione debba essere eseguito istantaneamente. In alcuni campi di elevata rilevanza finanziaria, ad esempio, questo dato di fatto può condurre a esiti assai divergenti in sede di applicazione delle leggi. L'articolo 370 capoverso 3 del Codice delle obbligazioni (CO; RS 220) prescrive che i difetti nascosti (od occulti) delle opere, ossia quelli non individuabili alla prima verifica destinata all'approvazione dell'opera dopo il suo ricevimento, devono essere segnalati non appena vengono scoperti, e quindi immediatamente: 
$\ll^{3}$ Ove i difetti si manifestassero soltanto più tardi, dovrà esserne dato avviso tosto che siano scoperti; altrimenti l'opera si ritiene approvata nonostante i difetti stessi.»

[105] Il Codice non specifica tuttavia entro quando la segnalazione deve essere fatta affinché il committente possa rifarsi sull'appaltatore. Le altre versioni linguistiche lasciano la stessa indeterminatezza:

$«^{3}$ Treten die Mängel erst später zu Tage, so muss die Anzeige sofort nach der Entdeckung erfolgen, widrigenfalls das Werk auch rücksichtlich dieser Mängel als genehmigt gilt.

${ }^{3}$ Si les défauts ne se manifestent que plus tard, le maître est tenu de les signaler à l'entrepreneur aussitôt qu' il en a connaissance; sinon, l'ouvrage est tenu pour accepté avec ces défauts.»

[106] La formulazione della disposizione non permette di inferire un termine ammissibile per notificare i difetti dopo la loro scoperta: «tosto che», «sofort nach»e «aussitôt que» sembrano indicare un'azione immediata, senza possibilità di attendere. Parrebbe tuttavia ragionevole che concretamente si avesse invece un'indicazione temporale assoluta o quantomeno di massima. Sulla questione ha infatti statuito il Tribunale federale precisando che gli avvisi devono essere dati al più tardi entro sette giorni dalla scoperta dei difetti (cfr. sentenza 4A_82/2008 del 29 aprile 2009, consid. 7.1). Nella prassi tale termine è tuttavia ritenuto problematico: essendo troppo breve, taluni committenti segnalano preventivamente difetti magari solo presunti, consapevoli che in seguito non sarebbe più possibile farlo (cfr. la motivazione dell'iniziativa parlamentare Hutter 12.502 «Termini di avviso più equi nel contratto di appalto»).

[107] Vista la giurisprudenza della Corte suprema, la disposizione del CO dovrebbe recitare piuttosto «senza indugio» («unverzüglich», «sans délai»), concedendo così un certo lasso di tempo per eseguire le necessarie verifiche di dettaglio e accertare i (presunti) difetti riscontrati. In questo caso non entrano in linea di conto eventuali considerazioni linguistiche d'ordine diacronico sullo scarto ultracentenario che ci separa dalla stesura del $\mathrm{CO}$ volte a relativizzare l'espressione «tosto che», di fatto variante desueta di «non appena».

[108] Per completezza segnalo che l'iniziativa parlamentare Hutter 12.502 menzionata sopra, cui hanno dato seguito le Commissioni degli affari giuridici nel 2013 e nel 2014, propone un termine di 60 giorni per segnalare i difetti nascosti. Visto che parallelamente il Consiglio federale ha integrato il termine di 60 giorni nell'avamprogetto di modifica del $\mathrm{CO}$ su incarico della mozione Fässler 09.3392 «Rafforzare i diritti dei committenti nell'eliminazione dei difetti di costruzione», in consultazione tra agosto e novembre 2020, l'attuazione dell'iniziativa Hutter, già differita più volte, è slittata ulteriormente. Nel CO vi sarà ad ogni modo, se le Camere lo vorranno, un termine esplicito che non lascia dubbi.

\section{L'indeterminatezza al vaglio delle autorità giudiziarie}

[109] Dall'indeterminatezza della locuzione «senza indugio» usata nella legislazione possono nascere contenziosi su cui è chiamato a pronunciare il giudice. In alcuni casi la giurisprudenza ha 
così precisato quanto il legislatore, talvolta probabilmente senza essere cosciente della possibile portata delle sue scelte, ha stabilito con una certa apertura all'apprezzamento di chi applica la legge. Due cause originate dal modo in cui è stato interpretato l'avverbio «unverzüglich», una a livello federale e l'altra a livello cantonale, esemplificano il rinvio del problema alle autorità giudiziarie.

\subsection{Giurisprudenza federale: la celerità nel $\mathrm{CO}$}

[110] La disposizione incriminata in questo caso è l'articolo 107 capoverso 2 CO:

$«^{2}$ Wird auch bis zum Ablaufe dieser Frist nicht erfüllt, so kann der Gläubiger immer noch auf Erfüllung nebst Schadenersatz wegen Verspätung klagen, statt dessen aber auch, wenn er es unverzüglich erklärt, auf die nachträgliche Leistung verzichten und entweder Ersatz des aus der Nichterfüllung entstandenen Schadens verlangen oder vom Vertrage zurücktreten.

${ }^{2}$ Se l'adempimento non avviene neppure entro questo termine, il creditore può nulladimeno richiedere l'adempimento ed il risarcimento del danno pel ritardo, ma invece di ciò, purché lo dichiari immediatamente, può rinunciare alla prestazione tardiva $\mathrm{e}$ pretendere il danno derivante dall'inadempimento oppure recedere dal contratto.

${ }^{2}$ Si l'exécution n'est pas intervenue à l'expiration de ce délai, le droit de la demander et d'actionner en dommages-intérêts pour cause de retard peut toujours être exercé; cependant, le créancier qui en fait la déclaration immédiate peut renoncer à ce droit et réclamer des dommages-intérêts pour cause d'inexécution ou se départir du contrat.»

[111] Nella sentenza 4A_141/2017 del 4 settembre 2017 (consid. 4.2) il Tribunale federale ha confermato che la dichiarazione di rinuncia, effettuata dopo tre mesi circa, non è stata fatta «unverzüglich» («Die Beurteilung der Vorinstanz, dass die Verzichtserklärung nach rund drei Monaten nicht «unverzüglich» i.S.v. Art. 107 Abs. 2 OR war, ist nicht zu beanstanden.»). Secondo la massima Corte, l'interpretazione di «unverzüglich» risulta dalla valutazione della situazione contrattuale concreta e dagli interessi dei contraenti («Was unter «unverzüglich» zu verstehen ist, ergibt sich aufgrund der Beurteilung der konkreten Vertragslage und der Parteiinteressen.»). Queste condizioni possono dunque determinare motivi oggettivi che ammettono un differimento, ma non di tre mesi. Il giudizio del Tribunale federale lascia intendere che i termini corrispondenti delle altre due versioni linguistiche, «immediatamente» e «(déclaration) immédiate», non siano stati giudicati conformi alla volontà del legislatore (infatti non ne tiene conto) e che sarebbero state più opportune le espressioni «senza indugio» e "sans délai». Se tre mesi sono giudicati troppi, uno o due avrebbero ancora corrisposto a «unverzüglich»? Forse sì, ma «immediatamente» e «immédiate» non lasciano certo pensare a una possibile dilazione di uno o due mesi - e tanto meno di tre -, cosa che invece sarebbe stata pensabile in presenza delle espressioni «senza indugio» e «sans délai». 


\subsection{Giurisprudenza cantonale: la celerità nel CPP}

[112] L'espressione all'origine della contestazione in questo caso è «ohne Verzug», sinonimo di «unverzüglich», usata nell'articolo 229 capoverso $1 \mathrm{CPP}$ :

«1 In der Hauptverhandlung werden neue Tatsachen und Beweismittel nur noch berücksichtigt, wenn sie ohne Verzug vorgebracht werden [...].

${ }^{1}$ Nel dibattimento nuovi fatti e nuovi mezzi di prova sono considerati soltanto se vengono immediatamente addotti $[\ldots]$.

${ }^{1}$ Les faits et moyens de preuve nouveaux ne sont admis aux débats principaux que s'ils sont invoqués sans retard [...].»

[113] Nel considerando 3.b) della decisione BO.2017.4 del 16 novembre 2017 il Tribunale cantonale di San Gallo spiega come si debba intendere la locuzione «ohne Verzug» e ricorda la prassi dottrinale e quella giurisprudenziale cantonale in materia:

«Ohne Verzug〉 (Art. 229 Abs. 1 ZPO) bedeutet 〈sofort〉 (Botschaft ZPO, S. 7341), d.h. 〈unverzüglich nach der Entdeckung〉 (Leuenberger, ZPO Komm., Art. 229 N 9). Die herrschende Lehre und die kantonale Rechtsprechung gehen dabei in der Regel von einer Zehntagesfrist seit Entdeckung aus, innert welcher die Noven eingebracht werden müssen (das Bundesgericht hat sich dazu soweit ersichtlich noch nicht verbindlich geäussert $[\ldots])$.»

[114] Il messaggio del Consiglio federale, chiamato in causa, dà come sinonimi «ohne Verzug» e «sofort»: «Für alle (zulässigen) Noven gilt jedoch, dass sie jeweils sofort vorzubringen sind [... ]» (BBl 2006 7221, in particolare 7341, corsivo originale). La versione italiana del messaggio (FF 2006 6593, in particolare 6713) usa l'avverbio «immediatamente», come nel Codice, mentre la versione francese (FF 2006 6841, in particolare 6949) recita «immédiatement», determinando così, al pari del tedesco, uno scarto semantico rispetto al testo di legge.

[115] Il giudice, in questo caso, si fonda dunque su quanto afferma il Consiglio federale nel messaggio a sostegno del CPP e sulla dottrina. Unendo tali riscontri, il Tribunale cantonale interpreta le espressioni «ohne Verzug» (legge), «sofort» (messaggio) e «unverzüglich» (dottrina) come sinonimi. I nuovi fatti e nuovi mezzi di prova devono essere addotti nel giro di dieci giorni, il che sembra confermare la bontà delle espressioni «ohne Verzug» e "sans retard», benché vengano relativizzate nel messaggio, e indicare l'inadeguatezza di «immediatamente», difficilmente interpretabile come concessione di un differimento, seppur massimo, di una decade.

\section{Conclusione}

[116] Nel linguaggio comune «senza indugio» è una variante di «subito»e «immediatamente»e appartiene a un registro più alto. Nel linguaggio giuridico «senza indugio» è riferito a un atto eseguibile all'istante soltanto se non vi sono motivi oggettivi che possano ritardarne l'esecuzione (p. es. una fase procedurale che deve concludersi, l'attesa di un documento, di un nulla osta o di 
una decisione, circostanze contingenti legate all'ubicazione momentanea di una persona, aspetti organizzativi oppure un periodo di riflessione di durata variabile a seconda delle circostanze specifiche). L'esecuzione è quindi subordinata all'esistenza di condizioni particolari che permettono o richiedono di ritardarla. In assenza di simili condizioni non è lecito attendere e l'atto va compiuto subito, immediatamente.

[117] Gli avverbi «immediatamente» e «subito» indicano invece nel linguaggio giuridico un atto eseguibile all'istante, senza condizionamenti o senza attendere altre fasi procedurali.

[118] Nella prassi della scrittura delle leggi questa distinzione non sembra tuttavia essere attuata sistematicamente e spesso «senza indugio» è variante equivalente di «immediatamente» e «subito». D'altro canto, in molti casi «immediatamente» ha in realtà il valore di «senza indugio» in quanto evidentemente l'atto in questione, per motivi contingenti e agevolmente riconoscibili, non può essere compiuto subito. Inoltre, spesso vi è difformità tra le versioni linguistiche nell'uso delle espressioni e incoerenza anche all'interno della stessa versione.

[119] Un ulteriore problema risiede nel fatto che non è dato sapere se e in quale misura il tedesco e il francese degli estensori dei testi della legislazione federale facciano la necessaria distinzione tra i due concetti e quindi li usino correttamente. Va dunque sottolineato che l'adeguatezza del loro uso va esaminata caso per caso in funzione della tempistica che si vuole esprimere e a prescindere, quindi, dalle espressioni usate nelle altre versioni linguistiche.

[120] Si può dunque proporre uno specchietto delle equivalenze di massima nelle lingue ufficiali della legislazione federale:

senza indugio $=$ unverzüglich $=$ sans délai

immediatamente $=$ sofort $=$ immédiatement

[121] Come si è visto dagli esempi riportati, a queste espressioni se ne affiancano altre come «subito», «prontamente», «seduta stante», «tosto che» (nonché la variante grafica «senz'indugio»), rispettivamente «ohne Verzug», «umgehend», «sans retard», «sans tarder», «aussitôt que», «tout de suite». A rigore, i termini non sono intercambiabili e occorrere prestare la massima attenzione all'uso dell'una o dell'altra espressione, in funzione del caso specifico, valutando se un atto ammette o richiede una dilazione o no.

Grovanni Bruno, Cancelleria federale, Servizi linguistici centrali, Divisione italiana, Bellinzona, e-mail: giovanni.bruno@bk.admin.ch.

\section{Riferimenti bibliografici}

Bernasconi, Paolo et al. (2010): Codice svizzero di procedura penale (CPP). Commentario, Zurigo/San Gallo.

Donatsch, Andreas et al. (2014): Kommentar zur Schweizerischen Strafprozessordnung (StPO), $2^{\mathrm{a}}$ ed., Zurigo.

EgGer, Jean-Luc/Grandi, Filippo (2008): Il nuovo Codice di procedura penale: un cantiere anche linguistico, in: LeGes 19 (2008) 1.

Egger, Jean-Luc (2019): A norma di (chi) legge. Peculiarità dell'italiano federale, Milano.

Jeanneret, Yvan et al. (2019): Code de procédure pénale suisse. Commentaire, $2^{\mathrm{a}}$ ed., Basilea.

RikLin, Franz (2014): StPO Kommentar. Schweizerische Strafprozessordnung mit JStPO, StBOG und weiteren Erlassen, $2^{\mathrm{a}}$ ed., Zurigo. 
Scolari, Adelio (2002): Diritto amministrativo. Parte generale, Cadenazzo/Bellinzona.

Snozzi, Alfredo (2015): Lessico giuridico. Italiano, tedesco, francese, Bellinzona/Basilea. 